\title{
Estudio sobre las variables relacionadas con la deserción escolar en centros de formación de personas adultas
}

Enviado: 17 de enero de 2020 / Aceptado: 11 de febrero de 2020

Publicado: 12 de julio de 2020

LUIS MIGUEL MATEOS TORO, Departamento de doctorando de la Universidad Internacional de la Rioja (UNIR), Universidad Internacional de la Rioja (UNIR), España. luismiguel.mateos475@comunidadunir.net

LAURA ABELLÁN ROSELLO Departamento de Área de Métodos Investigación y Diagnóstico en Educación, Universidad de Internacional de la Rioja, España. laura.abellan@unir.net

\section{RESUMEN}

Introducción. El abandono escolar en España tiene una elevada tasa de deserción y ostenta una elevada tasa de paro juvenil por ello la presente investigación trata de conocer en qué medida influye el centro educativo, los recursos en el aula, los profesores y la situación personal y familiar en la deserción escolar de estudiantes matriculados en centros para adultos.

Metodología. La muestra recogida durante el curso 2018/2019 estaba formada por 46 personas, 16 hombres $(34 \%)$ y 31 mujeres $(66 \%)$ con la distribución de la muestra por edades entre los 18 y los 70 años $(\mathrm{M}=37 ; \mathrm{SD}=24,13)$. Se empleó el cuestionario estandarizado sobre fracaso escolar en alumnado de educación secundaria de un centro de formación de personas adultas creado por los autores Sancho y Grau (2012). Para obtener los resultados se ha utilizado un

\section{ABSTRACT}

Study on the variables that are related to school dropout in adult training centers

Introduction. School dropout in Spain has a high dropout rate and has a high youth unemployment rate. In the present investigation, he tries to know to what extent the educational center, the resources in the classroom, the teachers and the personal and family situation influenced his school dropout in students enrolled in adult centers. Methodology. The sample collected during the 2018/2019 academic year consisted of 46 people, 16 men (34\%) and 31 women (66\%) with the distribution of the sample by ages between 18 and 70 years $(M=37 ; S D=24.13)$. The standardized questionnaire on school failure in secondary school students of an adult training center created by the authors Sancho and Grau (2012) was used. To ob- 
análisis no paramétrico de una muestra, además, se ha realizado una regresión lineal y estudios descriptivos tanto de frecuencias como descriptivos. También se han comparado las medias mediante ANOVA de un factor. Resultados. Los resultados revelaron que las variables recursos económicos para materiales, recursos en el aula, recursos económicos familiar para la compra de materiales, desarrollo de la actividad por parte del profesor, relación que existe entre el centro y las familias e instalaciones adecuadas para el alumnado, podrían influir en el abandono escolar de formación para adultos. Discusión. Se comentan las implicaciones de estos hallazgos para la investigación y la práctica psicoeducativa.

Palabras Clave: Educación Adultos, Educación Secundaria, Fracaso escolar, Abandono Escolar Educación Secundaria. tain the results, a non-parametric analysis of a sample has been used, in addition, a linear regression and descriptive studies of both frequencies and descriptive studies have been performed. The averages have also been compared using one-way ANOVA. Results. The results revealed that the variables economic resources for materials, resources in the classroom, family economic resources for the purchase of materials, development of the activity by the teacher, relationship between the center and families and adequate facilities for students, they could influence the abandonment of adult education. Discussion. The implications of these findings for research and psychoeducational practice are discussed.

Keywords: Adult Education, Higher Education, Academic failure, High School Dropouts.

\section{Introducción}

En el sistema educativo Español, existe un grave problema de abandono escolar, deserción escolar y absentismo escolar que necesita ser estudiada en profundidad (OECD, 2018). En España todavía un $21,5 \%$ de los jóvenes de 18 a 24 años de edad ni han completado estudios postobligatorios, ni están estudiando, una tasa que dobla la media de la UE-27, situada en el 12\% (Serrano y Soler, 2015).

Existe un problema generalizado de desmotivación en el alumnado que el profesorado no sabe cómo afrontar. Este estado caracterizado por el desinterés, la falta de implicación y esfuerzo por aprender, repercute negativamente en el clima del aula (socialización), así como en el rendimiento escolar, y, además, es una de las principales causas de burnout entre el profesorado (Doménech y Gómez-Artiga, 2010).

\subsection{La motivación}

La falta de motivación del alumnado es uno de los principales problemas con los que se enfrenta actualmente el profesorado (Doménech y Abellán, 2017) que trabaja en centros de educación secundaria para personas adultas. La motivación del alumnado influye en su rendimiento académico, por tanto, la desmotivación influye negativamente sobre su rendimiento académico y de sus compañeros (Garrido 2013). 


\subsubsection{Teoría clásica de la motivación de logro (Atkinson y Feather, 1966)}

Según la teoría clásica de la motivación de logro (Atkinson y Feather, 1966) son tres los componentes de la conducta para conseguir un logro: motivo de logro, expectativas de éxito y el incentivo que se tiene para conseguir el logro. Por lo tanto, el motivo del éxito está relacionado con las experiencias vividas relacionadas con los estudiantes en etapas anteriores de su desarrollo, que les llevan a sentir orgullo cuando alcanzan un éxito, o a sentir vergüenza cuando fracasan (Gollwitzer, 2018). De esta forma van creando una tendencia a la aspiración de tener éxito y evitando el fracaso y del conflicto entre ambas situaciones surge la motivación de logro (Ames, 1992; Dweck y Legget, 1988; Nicholls, 1989). Por otro lado, las expectativas de éxito son los reconocimientos que el estudiante tiene sobre la probabilidad de realizar una tarea con éxito (Naranjo, 2009)

\subsubsection{Teoría de orientación a la meta (Maehr, 1984)}

La teoría de la orientación de la meta (Maehr, 1984), se puede definir desde un punto de vista clásico, normativo, multidimensional o de teoría reformulada, como indica Fuente (2004). La teoría clásica de orientación a la meta indica que hay dos tipos de metas, las metas académicas y las metas sociales. Las metas académicas, se refieren a los motivos en lo que se encuentran los estudiantes (Dörnyei, 2000). Y se subdividen en tres: Metas de aprendizaje, el objetivo de los estudiantes es obtener el conocimiento de forma eficaz. Metas de rendimiento, el objetivo es conseguir realizar la tarea mejor que los demás, por lo tanto, se centra en una habilidad. Metas centradas en el yo, el estudiante se compara con los demás para hacerse un poco una idea de cómo está realizando la tarea. Por otro lado, se trata el grado de incentivo que tiene un estudiante para alcanzar el éxito en un momento concreto con lo que supone un desafío que supone una relación inversa con las expectativas del éxito.

Por consiguiente, las metas sociales se refieren a aquello que estimula a los estudiantes de una forma o de otra con los demás en el ámbito académico. Estudios realizados por Wenrzel y Wigfield (1998) avalan la importancia de este tipo de metas. Por su parte, la teoría reformulada de la orientación de una meta agrega ciertos pensamientos a la teoría clásica. Uno de ellos se refiere a que las metas de rendimiento no tienen que ser des-adaptativas, siempre que el alumnado obtenga sus metas de aprendizaje (Elliot, 1997).

El estudiante que muestre ambos tipos de metas obtendrá un efecto positivo en cuanto al desempeño de sus tareas (Pintrich, 2000).

\subsubsection{La motivación en la etapa escolar}

Una de las razones significativas de la incidencia de la motivación en el aprendizaje escolar es la falta de interés por el estudio y la actitud desfavorable hacia un aprendizaje al que no terminan de verle 
la utilidad (Doménech-Betoret, Gómez-Artiga y Lloret-Segura, 2014) lleva al estudiante a la desmotivación, la dejadez o incluso el abandono. Así, en la etapa de educación secundaria, se puede encontrar al alumnado que pasa por momentos de crisis pero que obligatoriamente deben estar en la escuela. Y esto les lleva en ocasiones a acabar no queriendo estar en ella (Funes, 2010).

La transición de la educación primaria a la educación secundaria les obliga a estar en continua adaptación, es una etapa que dejan de ser niños sin llegar a ser adultos (Irsona, 2013), por lo que les resulta complejo encontrar su lugar y su identidad. A todo esto, habría que añadir que los estudiantes que obtuvieron resultados negativos en el último curso de primaria se enfrentan a secundaria con mayores niveles de estrés, lo cual hace que su motivación al comienzo de la nueva etapa sea también menor (Harten, Rumbaugh y Kowalski 1992). Esto provoca que dichos estudiantes entren ya desmotivados y algunos incluso mantienen esta desmotivación a lo largo de los años.

Esta situación se ve agravada en la edad adulta, por lo que los alumnos matriculados en escuelas de adultos para obtener el certificado obligatorio de escolaridad se muestran con un nivel de desmotivación elevado, como se expone en el estudio realizado por Forstmeier y Maercker (2008).

\subsubsection{La motivación intrínseca y desmotivación intrínseca}

Desde esta perspectiva la motivación del alumnado influye en su rendimiento académico, por tanto la desmotivación influyen negativamente sobre su rendimiento académico y de sus compañeros en el cual según:

Abraham Maslow (1943) describe que cuando una persona se siente desmotivada es debido a que no ha cubierto una serie de necesidades inferiores. Subdivide las necesidades humanas en cinco categorías, necesidades básicas, necesidades de seguridad, necesidades sociales, necesidades afectivas y autorrealización.

\subsection{La Socialización}

\subsubsection{Teoría Sociocultural de Vigotsky (1978)}

Según esta teoría se define el desarrollo de las personas que están relacionadas directamente con la interacción en el contexto en el que viven, es decir el ser humano toma como suyos los signos pertenecientes a la cultura que le envuelve y posteriormente los interioriza, así como desarrolla la teoría sociocultural a finales de los años veinte, indicando que para conocer a una persona es necesario conocer también su vida y las condiciones que le rodean (Sanz, 2015). 
Para el autor, la ley genética general del desarrollo psíquico, donde para que las funciones del niño aparezcan en el plano psicológico, es necesario que aparezcan anteriormente en el plano social, se trata del momento más importante en el desarrollo del niño dado que es cuando el lenguaje se unen con las actividades que se lleva a cabo, ya que es entonces cuando la palabra y la acción empiezan a verse relacionados y el niño interactúa con su entorno a través del lenguaje adquirido (Harry, 2003).

Otro concepto importante es el de Zona de Desarrollo Próximo (ZDP), basada en que cada persona tiene un nivel de desarrollo real, aquello que puede aprender sin necesidad de la ayuda de los demás y un nivel de desarrollo potencial (Hernández, 1997; Apple, 1997 y Tamarit, 1997). Se refiere a aquel aprendizaje que puede ser adquirido por mediación de personas más capaces, produciéndose el desarrollo hasta alcanzar el máximo nivel potencial. Por ello, para alcanzar el nivel potencial de un estudiante es necesario la colaboración de otras personas implicadas en su aprendizaje y por tanto la socialización de todo el proceso de aprendizaje-enseñanza se convierte en el resultado de la relación del estudiante con el mundo que le rodea (Vigotsky, 2005; Coll, 1991).

\subsubsection{Teoría de Aprendizaje Social de Bandura}

La teoría de Aprendizaje Social desarrollada por Bandura (1977), describe cómo las personas adquieren nuevos aprendizajes a través de la observación de otras personas, el elemento social se da lugar en el sitio donde los individuos aprenden y desarrollan nuevas conductas. Se puede diferenciar dos tipos de aprendizajes, el aprendizaje a través de las consecuencias y el aprendizaje por imitación.

Teniendo en cuenta el aprendizaje social, en la edad adulta se puede hablar de "autoeficacia o expectativa de éxito" que se definen como la creencia de un individuo en su propia capacidad para realizar una tarea determinada; mientras que "la expectativa de resultados" se define como la creencia de que esforzarse dará lugar a un resultado deseado. Los estudiantes con fuertes creencias de autoeficacia suelen visualizar escenarios de éxito que proporcionan recursos de apoyo orientados a mejorar su rendimiento académico (Bandura, 1993). Por lo tanto, es probable que estos alumnos construyan creencias anticipatorias más positivas sobre los resultados esperados teniendo en cuenta lo que aprenden del resto del alumnado que les rodea (Doménech-Betoret, Abellán-Roselló, Gómez-Artiga, 2019).

\subsubsection{La socialización en la etapa escolar}

La adolescencia es la etapa que abarca la transición de la niñez a la adultez. Teniendo esto en cuenta, la socialización es el proceso por el cual el niño adquiere conocimientos, valores, costumbres y sentimientos que influyen en la adaptación a un comportamiento social positivo (Musitu, 2000). Por lo tanto, 
socializar se trata de un proceso a través del cual el niño aprende a diferenciar los comportamientos positivos de los negativos, aunque este proceso de socialización comienza en la niñez, continúa hasta la adolescencia, etapa donde se hacen más relevante otros ámbitos diferentes ámbitos familiares, grupo de iguales, el ámbito escolar o los medios de comunicación (Bandura, 2001). Por lo cual, los adolescentes suelen prestan mayor atención a la familia en ciertos temas, como cuestiones morales, económicas o educativas, mientras que dan mayor credibilidad a los temas iguales en temas como la amistad, las relaciones o el tiempo libre (Smetana, 1993).

Por otro lado, la conducta prosocial ofrece al adolescente cierto bienestar, mientras que la conducta agresiva o una conducta antisocial produce el rechazo de los demás. La importancia de este aspecto reside fundamentalmente en que los adolescentes aceptados por sus iguales, presentan una adaptación más favorable tanto en el ámbito social, como en el personal y el académico (Chen, 2006).

La educación de adultos se fundamenta en la educación permanente y continuada. Este programa está regulado y está dentro del sistema educativo español a través del decreto 159/2002, de 28 de mayo por el que se aprueba el reglamento orgánico de los institutos provinciales de formación para adultos.

\subsubsection{La socialización en la adolescencia}

La adolescencia es la etapa que abarca la transición de la niñez a la adultez.

La socialización es el proceso por el cual el niño adquiere conocimientos, valores, costumbres y sentimientos que influyen en la adaptación a un comportamiento social positivo (Musitu 2000).

Por lo tanto, socializar se trata de un proceso a través el niño aprende a diferenciar los comportamientos positivos de los negativos, aunque este proceso de socialización comienza en la niñez, continúa hasta la adolescencia, etapa donde se hacen más relevante otros ámbitos diferentes ámbito familiar, grupo de iguales, el ámbito escolar o los medios de comunicación (Bandura, 2001).

Por lo cual, los adolescentes suelen prestan mayor atención a la familia en ciertos temas, como cuestiones morales, económicas o educativas, mientras que dan mayor credibilidad a los temas iguales en temas como la amistad, las relaciones o el tiempo libre (Smetana, 1993).

Por otro lado, la conducta pro social ofrece al adolescente cierto bienestar, mientras que la conducta agresiva o una conducta antisocial produce el rechazo de los demás. La importancia de este aspecto reside fundamentalmente en que los adolescentes aceptados por sus iguales, presentan una adaptación más favorable tanto en el ámbito social, como en el personales y el académico (Chen, 2006). 
Lo que se llega a la conclusión, la conducta pro social se desarrolla el adolescente con sus iguales lo que se convierte en un factor de protección ante posibles problemas de conducta.

\subsection{Objetivos}

El objetivo principal de este estudio es conocer en qué medida influye el centro educativo, los recursos en el aula, profesores que imparten clase, situación personal y familiar en la deserción escolar de estudiantes que están cursando Educación obligatoria en centros de adultos. De este objetivo se derivan dos específicos. Por un lado, desde la perspectiva motivacional poder conocer el motivo de la reintegración escolar del alumno conociendo los motivos de su abandono escolar y cómo influye el centro educativo en el que estudia, el aula y los recursos que disponibles en el aula, la relación con los profesores, el desarrollo de las clases con los profesores y la situación personal y familiar del alumno cuando se produjo su deserción.

Por otro lado, desde la perspectiva relacionado con la socialización poder comprender el motivo de su deserción escolar conociendo como influye los medios económicos familiares para la compra de materiales, el desarrollo de la actividad por parte del profesor respeta los diferentes ritmos de aprendizaje de los alumnos, la relación que existe entre el centro y las familias y las instalaciones adecuadas para el alumnado.

\section{MATERIAL Y MÉTODO}

\subsection{Tipo de estudio}

El tipo de estudio es cuantitativo siendo una investigación cuasi experimental con una muestra no aleatorizada y transversal. Además, este estudio se enmarca dentro de los procesos de revisión y elaboración de instrumentos psicométricos, partiendo de una propuesta metodológica inmensa en la medición educativa. Las características que han guiado la investigación han sido propuestas por Jornet y Suarez (1989) para la construcción de un test normativo.

\subsection{Participantes}

La muestra está compuesta de 47 personas que están matriculadas en educación para adultos para obtener el título de Educación Secundaria Obligatoria. 16 hombres siendo el 34\% de la muestra y por 31 mujeres con el porcentaje de $66 \%$, con edades que oscilan de 18 años a 70 años con una edad media de 37 años y con una desviación típica de 24,13. 


\subsection{Instrumentos}

Para realizar esta investigación de campo, se ha utilizado el cuestionario sobre fracaso escolar en alumnado de Educación Secundaria de un centro de formación de personas adultas elaborado por Sancho y Grau (2013) quienes los autores lo consideran como una idea de fracaso así como un tema multidimensional.

El cuestionario mencionado trata de 35 reactivos, 26 de los cuales son valorados con una escala de puntuación compuesta por las opciones de muy insatisfecho, insatisfecho, satisfecho y muy satisfecho. Las cinco cuestiones restantes están configuradas como preguntas abiertas. Por otro lado, los 31 ítems se han dividido por bloques, en el primer bloque se hace referencia al centro educativo, en el segundo al aula, el tercero al profesorado, el cuarto bloque hace referencia al profesorado, el quinto bloque hace referencia a la situación personal y familiar, el último bloque hace referencia el motivo de su retorno al sistema educativo.

Respecto a los indicadores de fiabilidad los autores han analizado el coeficiente de alfa de Cronbath añadiendo el estadístico total-elemento para obtener la consistencia interna de forma global. Los resultados fueron positivos según los análisis giran en torno a un porcentaje aceptable de consecuencia interna de .756 según el modelo de Cronbath.

\section{Resultados}

En un primer análisis se analizan las variables de los recursos económicos para materiales necesarios para el centro educativo con respecto a la situación personal y familiar, los servicios complementarios con respecto al centro educativo y los recursos en el aula con respecto a la escala respecto al aula.

\subsection{Regresión lineal motivacional}

En la Tabla 1 se recogen el análisis de regresión de las distintas variables observando que el valor de $R$ es 0.362 por que se trata de un valor significativo, el $r$ cuadrado valor superior a $>0$ existiendo significación, con respecto al R cuadrado ajustado también posee valores significativos de 0.62 superando los mínimos establecidos, con respecto al error estándar de la estimación existe un valor significativo y esto sin embargo se trata de un hándicap dado que existe desviación lineal siendo el valor de 0.453

Tabla 1. Regresión lineal.

\begin{tabular}{|c|c|c|c|c|}
\hline Modelo & R & R cuadrado & R cuadrado ajustado & $\begin{array}{c}\text { Error estándar } \\
\text { de la estimación }\end{array}$ \\
\hline 1 &, $362^{\mathrm{a}}$ &, 131 &, 062 &, 453 \\
\hline
\end{tabular}




\subsection{ANOVA motivacional}

En la tabla 2 se utiliza como variable dependiente el sexo y como variables independientes los recursos económicos para materiales, los servicios complementarios y los recursos en el aula. Se observa que en la suma de cuadrados existe un importante valor acerca de los residuos con un valor mayor que la regresión, ambas medias cuadradas tienen un valor significativo con 0.392 y 0.205 con respecto al modelo de regresión y con los residuos respectivamente, el valor de F es de 1,910 y la significación es de 0.144 siendo superior a 0.05 por lo que este modelo se asemeja con mayor precisión como se observar en los resultados.

Tabla 2. Anova.

\begin{tabular}{|c|c|c|c|c|c|c|}
\hline \multicolumn{2}{|c|}{ Modelo } & $\begin{array}{c}\text { Suma de } \\
\text { cuadrados }\end{array}$ & gl & $\begin{array}{c}\text { Media } \\
\text { cuadrática }\end{array}$ & F & Sig. \\
\hline \multirow{2}{*}{1} & Regresión & 1,176 & 3 &, 392 & 1,910 & \\
\cline { 2 - 7 } & Residuo & 7,800 & 38 & ,205 & \\
\hline
\end{tabular}

\subsection{Coeficientes motivacionales}

En la tabla 3 se observar los coeficientes no estandarizados con el conjunto de las variables analizadas, los servicios complementarios, los recursos en el aula y los recursos económicos para materiales en su conjunto tiene un valor significativo de 2,671 teniendo valores negativos como ocurre en los servicios complementarios y los recursos económicos para materiales y valores positivos con respecto a los recursos en el aula.

Existe significación individualmente y en todas significativas siendo 0.727 en los recursos en el aula, 0,341 para los recursos económicos para materiales y sin embargo grupalmente no tienen significación al tener un valor de 0,00 .

En la tabla 4 se analiza los residuos de la distribución que posee una desviación estándar significativa con el valor de 0.169 con respecto al valor pronosticado siendo este dato superior con respecto al residuo con el valor de 0.436 .

El valor pronosticado estándar tiene una desviación estándar de 1 siendo el máximo pronosticado y el residió estándar se acerca al valor al poseer el valor de 0.963 . 
Tabla 3. Coeficientes.

\begin{tabular}{|c|c|c|c|c|c|c|}
\hline & \multirow[b]{2}{*}{ Modelo } & \multicolumn{2}{|c|}{$\begin{array}{c}\text { Coeficientes } \\
\text { no estandarizados }\end{array}$} & \multirow{2}{*}{$\begin{array}{c}\text { Coeficientes } \\
\text { estandarizados } \\
\text { Beta }\end{array}$} & \multirow[b]{2}{*}{$\mathbf{T}$} & \multirow[b]{2}{*}{ Sig. } \\
\hline & & B & $\begin{array}{c}\text { Error } \\
\text { estándar }\end{array}$ & & & \\
\hline \multirow[t]{4}{*}{1} & (Constante) & 2,671 &, 557 & & 4,797 &, 000 \\
\hline & $\begin{array}{c}\text { Servicios } \\
\text { Complementarios }\end{array}$ &,- 204 &, 100 &,- 323 & $-2,044$ & ,048 \\
\hline & Recursos en el aula & 045 &, 127 & ,061 &, 352 & ,727 \\
\hline & $\begin{array}{l}\text { Recursos económi- } \\
\text { cos para materiales }\end{array}$ &,- 172 & ,178 &,- 163 &,- 964 & ,341 \\
\hline
\end{tabular}

Tabla 4. Resumen de los residuos.

\begin{tabular}{|c|c|c|c|c|c|}
\hline & Mínimo & Máximo & Media & Desviación estándar & N \\
\hline Valor pronosticado & 1,35 & 2,09 & 1,69 &, 169 & 42 \\
\hline Residuo & $-1,086$ &, 493 &, 000 & 42 \\
\hline $\begin{array}{c}\text { Valor pronosticado } \\
\text { estándar }\end{array}$ & $-2,025$ & 2,335 &, 000 & 1,000 & 42 \\
\hline Residuo estándar & $-2,397$ & 1,089 &, 000 &, 963 & 42 \\
\hline
\end{tabular}

\subsection{Regresión lineal socialización}

En la tabla 5 se observar que la tanto el valor de R como el R cuadrado tienen valores significativos con 0.190 y 0.063 respectivamente, sin embargo, el valor de $\mathrm{R}$ cuadrado ajustado tiene un valor negativo con 0.063 y el error estándar de la estimación tiene un valor de 0,476 existe un valor importante del efecto de la variable queda hándicap con el análisis utilizado.

Tabla 5. Regresión lineal.

\begin{tabular}{|c|c|c|c|c|}
\hline Modelo & R & R cuadrado & R cuadrado ajustado & $\begin{array}{c}\text { Error estándar } \\
\text { de la estimación }\end{array}$ \\
\hline 1 &, $190^{\text {a }}$ &, 036 &,- 063 &, 476 \\
\hline
\end{tabular}




\subsection{ANOVA socialización}

En la tabla 6 se ha realizado un análisis de ANOVA con el modelo de regresión obtiene un valor significativo de 0.329 y con respecto a los residuos se obtiene significativo con el valor de 8.830 , en la media cuadrática existen valores de 0.082 y 0.226 con respecto al modelo de regresión y al modelo de residuos respectivamente. La significación es de 0.833

Tabla 6. ANOVA.

\begin{tabular}{|c|c|c|c|c|c|c|}
\hline \multicolumn{2}{|c|}{ Modelo } & $\begin{array}{c}\text { Suma de } \\
\text { cuadrados }\end{array}$ & gl & $\begin{array}{c}\text { Media } \\
\text { cuadrática }\end{array}$ & F & Sig. \\
\hline \multirow{2}{*}{1} & Regresión &, 329 & 4 &, 082 &, 364 & \\
\hline & Residuo & 8,830 & 39 &, 226 & & \\
\cline { 2 - 7 } & Total & 9,159 & 43 & & \\
\hline
\end{tabular}

\subsection{Coeficientes socialización}

En la tabla 7 se puede observar que en los coeficientes no estandarizados tienen valores negativos en todas las variables excepto en el desarrollo de la actividad en la clase respeta los diferentes ritmos de aprendizaje de los alumnos. El error estándar de los coeficientes no estandarizados es significativo en todas las variables.

Tabla 7. Coeficientes.

\begin{tabular}{|c|c|c|c|c|c|c|}
\hline \multirow{2}{*}{\multicolumn{2}{|c|}{ Modelo }} & \multicolumn{2}{|c|}{$\begin{array}{l}\text { Coeficientes no } \\
\text { estandarizados }\end{array}$} & \multirow{3}{*}{$\begin{array}{c}\text { Coeficientes } \\
\text { estandarizados } \\
\text { Beta }\end{array}$} & \multirow{3}{*}{$\begin{array}{c}\mathbf{T} \\
3,484\end{array}$} & \multirow{3}{*}{$\begin{array}{l}\text { Sig. } \\
, 001\end{array}$} \\
\hline & & \multirow{2}{*}{$\begin{array}{c}\mathbf{B} \\
2,229\end{array}$} & \multirow{2}{*}{$\begin{array}{c}\begin{array}{c}\text { Error } \\
\text { estándar }\end{array} \\
\text {,640 }\end{array}$} & & & \\
\hline 1 & (Constante) & & & & & \\
\hline & $\begin{array}{l}\text { Recursos económicos } \\
\text { para materiales }\end{array}$ &,- 063 &, 175 &,- 065 &,- 357 & ,723 \\
\hline & $\begin{array}{c}\text { Respeta diferentes ritmos } \\
\text { de aprendizaje }\end{array}$ & ,022 &, 189 & ,024 &, 115 & ,909 \\
\hline & $\begin{array}{l}\text { Relación familia } \\
\text { profesorado }\end{array}$ &,- 113 &, 148 &,- 163 &,- 765 & ,449 \\
\hline & Instalaciones adecuadas &,- 013 &, 127 &,- 019 &,- 102 & ,919 \\
\hline
\end{tabular}


Existe una significación individualmente de todas las variables, pero en el conjunto de las variables no existe una significación significativa.

En la tabla 8 se puede observar de las estadísticas de los residuos, los valores oscilan desde el mínimo con valores negativos y el máximo con valores positivos. Existe una elevada desviación estándar con respecto el residuo de la muestra.

Tabla 8. Estadísticas de Residuos.

\begin{tabular}{|c|c|c|c|c|c|}
\hline & Mínimo & Máximo & Media & $\begin{array}{c}\text { Desviación } \\
\text { estándar }\end{array}$ & N \\
\hline Valor pronosticado & 1,56 & 1,97 & 1,70 &, 088 & 44 \\
\hline Residuo &,- 791 &, 438 &, 000 &, 453 & 44 \\
\hline $\begin{array}{c}\text { Valor pronosticado es- } \\
\text { tándar }\end{array}$ & $-1,632$ & 3,005 &, 000 & 1,000 & 44 \\
\hline
\end{tabular}

\section{Discusión}

Tras la realización de los dos bloques de análisis se puede concluir que, por un lado, la variable referida a motivacional, se puede observar que los recursos económicos para materiales puede predecir la deserción escolar con una puntuación significativa de .341. Existiendo la relación más alta de la variable recursos en el aula con una puntuación de .727. que puede ayudar a mejorar la motivación del alumnado.

Por otro lado, la variable referida a socialización, tras la indagación realizada se puede comprobar que los recursos económicos para materiales con una puntuación significativa de .723. así como respetar los diferentes timos de aprendizaje con una puntuación significativa de .909. del modo que la relación de la familia con el profesorado con una puntuación significativa de .449. de forma que las instalaciones adecuadas para las necesidades del alumnado obtiene una puntuación significativa de .919 . siendo la puntuación más alta, ayudando a como puede mejorar la socialización del alumnado en el abandono escolar.

\section{Conclusiones}

Los recursos económicos disponibles por las familias, incluye la función de supervivencia escolar la probabilidad acumulada de que una persona haya salido de la escuela. A los 12 años, sólo 1.7\% de los 
adolescentes desertaron, hacia los 14 años, ya $7.6 \%$ de los jóvenes habían salido del sistema escolar. A partir de los 15 años la deserción se incrementó notablemente, pues a la edad de 15 años $19.2 \%$ de los adolescentes habían desertado la escuela y esta probabilidad acumulada se elevó a 36\% para los 17 años de edad. (Vargas Valle y Valadez García, 2016), coincidiendo con los datos obtenidos.

El profesor no respeta los diferentes ritmos de aprendizaje de los alumnos como indica el autor en su estudio, el papel del profesor es determinante en el abandono del alumno (Butti, 2018).

Los padres y madres españoles conocen mejor el centro educativo de sus hijos, participan en mayor medida en la vida del mismo, contribuyen más al desarrollo del aprendizaje del alumnado desde el hogar, y poseen relaciones más positivas con el centro que las familias inmigrantes (Prados et al., 2016).

La realidad de las escuelas portuguesas y la necesidad de implementar vías de aprendizaje alternativas, presenta un estudio sobre el potencial de las TIC para crear un modelo inclusivo de reversión del fracaso en los estudiantes que están en problemas con la escuela y en riesgo de abandono escolar temprano (Sanches, 2017), coincidiendo con los recursos en el aula y los servicios complementarios en el centro.

El presente estudio resulta innovador, puesto que se han encontrado escasos estudios donde se trabaje con población adulta estudiando Educación Secundaria Obligatoria. Como limitación, la muestra podría haber sido más amplia, llegando a otras comunidades, o incluyendo otras variables relacionadas. Futuras investigaciones, deberían tener en cuenta un estudio más extenso para valorar si las variables estudiadas continúan siendo significativas a lo largo del tiempo y añadir otras variables que la literatura científica también aconseja trabajar.

Es esencial que el profesorado que trabaja con alumnado con estas características, estén al día y colaboren con estas investigaciones, ya que les proporcionan información relevante sobre estudiantes con alta probabilidad de deserción y fracaso escolar. Asimismo, es importante que proporcionen un ambiente de confianza y conocimiento para que sus estudiantes se sientan seguros en el desarrollo del aprendizaje y logren alcanzar la titulación cursada. Por último, estos resultados pueden ser útiles para contribuir a detectar dificultades y realizar actuaciones preventivas.

\section{Referencias}

Apple, W. M. (1997). Teoría crítica y educación. 1ª Edición. Buenos Aires; Editorial Niño y Dávila Editores.

Bandura, A. (2001). Social cognitive theory: an agentic perspective. Annual Review of Psychology, $52,1-26$. 
Butti, F. (2018). Las condiciones del éxito y del fracaso escolar. Un estudio de representaciones sociales y prácticas pedagógicas. Nordeste, 0(15), 97-107.

Chen, K. (2006). Social skills intervention for students with emotional/behavioural disorders: a literature review from the american perspective. Educational Research and Reviews, 1(3), 143-149.

Dörnyei, Z. (s. f.). Motivation in action: Towards a process-oriented conceptualisation of student motivation I Kopernio. Recuperado 23 de febrero de 2020, de https://kopernio.com/ viewer?doi=10.1348/000709900158281\&token=WzE3NDE4NzcsIjEwLjEzNDgvMD AwNzA5OTAwMTU4MjgxIl0.Mq7il3MS-0PubfJ11jDWv8XMJKU

Doménech, F. (2018). The Educational Situaction Quality Model: Recent Advances. Front. Psychol. DOI: https://doi.org/10.3389/fpsyg.2018.00328

Doménech, F., Gómez-Artiga, A., Lloret, S. (2014). Personal variables, motivation and avoidance learning strategies in undergraduate students. Learning and Individual Differences, 2014, vol. 35, pp. 122-129, DOI: 10.1016/j.lindif.2014.06.007

Doménech-Betoret, F., Gómez-Artiga, A., Abellán-Roselló, L. (2019). The Educational Situation Quality Model: A New Tool to Explain and Improve Academic Achievement and Course Satisfaction. Front. Psychol. 10:1692. doi: 10.3389/fpsyg.2019.01692

Harter, S., Rumbaugh, N. y Kowalski, P. (1992). Individual Differences in the Effects of Educational Transitions on Young Adolescent's Perceptions of Competence and Motivational Orientation. American Educational Research Journal, 29(4), 777-807.

Harry, D. (2003). Vigotsky y la pedagogía. 1ª Edición. México; Editorial Paidós.

Hernández, G (1997) Módulo Fundamentos del Desarrollo de la Tecnología Educativa (Bases Psicopedagógicas). Coordinador: Frida Díaz Barriga Arceo. México: Editado por ILCEOEA.

Elliot, A. J. (1997). Integrating the "classic" and "contemporary" approaches to achievement motivation: A hierarquical model of approach and avoidance achievement motivation. En Maerh y P.R. Pintrich (Eds.), Advances in motivation and achievement. Greenwich, CT: JAI Press.

Enguita, M. F. (s. f.). DEL DESAPEGO AL DESENGANCHE Y DE ÉSTE AL FRACASO. $4,15$. 
Folgar, M. I., Rey, C. N., \& Lamas, M. F. (2013). La transición de la Educación Primaria a la Educación Secundaria: Sugerencias para padres. Innovación educativa, (23). https://doi. org/10.15304/ie.23.642

Forstmeier, S. y Maercker, A. (2008). Motivational Reserve: Lifetime Motivational Abilities Contribute to Cognitive and Emotional Health in Old Age. Psychology and Aging, 23(4), 885-899. DOI: $10.1037 / \mathrm{a} 0013602$

Funes, J. (2010). 9 ideas clave. Educar en la adolescencia. Barcelona: Graó.

Gollwitzer, P. M. (2018). Goal Concept: A Helpful Tool for Theory Development and Testing in Motivation Science. Motivation Science, 4 (3), 185-205. http://dx.doi.org/10.1037/ $\underline{\operatorname{mot} 0000115}$

Maslow, A. H. (1991). Motivación y personalidad. Ediciones Díaz de Santos.

Misitu, G. (2000) Socialización familiar y valores en el adolescente. Un análisis intercultural Recuperado el 18/06/2019 https://www.google.com/url?sa=t\&rct=j\&q=\&esrc=s\&source =web\&cd=1\&cad=rja\&uact=8\&ved=2ahUKEwir9Ie8jsnjAhVJ1 xoKHV9aARgQFjA AegQIABAC\&url=https\%3A\%2F\%2Fwww.raco.cat\%2Findex.php\%2FAnuarioPsico logia\%2Farticle\%2Fdownload\%2F61542\%2F88397\&usg=AOvVaw31FNYqMcdBd He ej23Z3fT

Naranjo Pereira, M. L. (2009). Motivación: Perspectivas teóricas y algunas consideraciones de su importancia en el ámbito educativo. Revista Educación, 33(2), 153. https://doi. org/10.15517/revedu.v33i2.510

OECD. (2018). Programme for international students assessment (PISA). Results from PISA 2018. Recuperado de: https://www.educacionyfp.gob.es/inee/evaluaciones-internacionales/ pisa/pisa-2018.html

Pintrich, P. R. (1989). The dynamic interplay of student motivation and cognition in the college classroom. En C. Ames \& M. Maehr (Eds.), Advances in motivation and achievement. Motivation enhancing environments. Greenwich, CT: JAI Press.

Prados, M. ${ }^{a}$ Á. H., Vicente, M. ${ }^{a}$ Á. G., Martínez, J. P., \& Sanz, M. ${ }^{a}$ P. G. (2016). Familia, Inmigración Y Comunicación Con El Centro Escolar: Un Estudio Comparativo. Educación XX1, 19(2), 127-151. 
Sancho Álvarez, C., Grau Vidal, Roser (2013) Diseño y validación de un cuestionario sobre fracaso escolar en alumnado de educación secundaria de un centro de formación de personas adultas, volumen 13 Recuperado el 18/06/2019 https://revistas.ucr.ac.cr/index.php/aie/ article/view/11716

Serrano Martínez, L. and Soler Guillén, Á. (2015). Economía y SociedadInformes 2015Economía y SociedadInformes Recuperado el 15/06/2019 https://www.fbbva.es/wp-content/ uploads/2017/05/dat/DE 2015 formacion_y empleo.pdf

Smetana, J.G. (1993). Conceptions of parental authority in divorced and married mothers and their adolescents. Journal of Research on Adolescence, 3, 19-39.

Vargas Valle, E. D., \& Valadez García, A. (2016). Calidad de la escuela, estatus económico y deserción escolar de los adolescentes mexicanos. Revista electrónica de investigación educativa, 18(1), 82-97.

Vigotsky, L. S. (1978). Pensamiento y lenguaje. $3^{a}$. Edición. México; Editorial Paidós.

Vigotksy, L.S (2005) El desarrollo de los procesos psicológicos superiores. $2^{a}$ edición. Barcelona: Grijalbo.

Wentzel, K. R., \& Wigfield, A. (1998). Academic and social motivational influences on students' academic performance. Educational Psychology Review, 10(2), 155-175. https://doi. org/10.1023/A:1022137619834 